\title{
"Sandwich" Technique of Total Urethral Reconstruction in the Laparoscopic Radical Prostatectomy: A Prospective Study
}

This article was published in the following Dove Press journal: Cancer Management and Research

\author{
Yong Liu ${ }^{1,2}$ \\ Qinxin Zhao ${ }^{3}$ \\ Feiya Yang ${ }^{3}$ \\ Mingshuai Wang $\mathbb{D}^{\prime}$ \\ Nianzeng Xing ${ }^{1,3}$ \\ 'Department of Urology, Capital Medical \\ University, Beijing Chaoyang Hospital, \\ Beijing, I0002I, People's Republic of \\ China; ${ }^{2}$ Department of Urology, Weihai \\ Municipal Hospital, Weihai, 264200, \\ People's Republic of China; ${ }^{3}$ Department \\ of Urology, National Cancer Center/ \\ National Clinical Research Center for \\ Cancer/Cancer Hospital, Chinese \\ Academy of Medical Sciences and Peking \\ Union Medical College, Beijing, I0002I, \\ People's Republic of China
}

Background: Early incontinence that has great impact on the quality-of-life is one usual drawback after laparoscopic radical prostatectomy (LRP). This prospective study aims at further documenting the improved effect of the "Sandwich" urethra reconstruction technique on continence at the early stage after LRP.

Methods: During the period from October 2017 to December 2018, 130 patients undergoing LRP in our institution were recruited into this prospective study. Sixty-six patients in Group A received LRP with the "Sandwich" technique of urethra reconstruction, while the remaining 64 patients in Group B did not adopt this reconstruction technique. The basic clinical data, perioperative related data, urinary continence, and urodynamic tests were analyzed and evaluated.

Results: There is no statistical difference in patients' basic clinical data, perioperative related data except urethral reconstruction time, which was $23.49 \pm 4.72$ minutes in Group A and 20.16 \pm 5.75 minutes in Group B $(P<0.001)$. The continence rates in Group A at 2, 4, 8, and 12 weeks were $54.55 \%, 83.33 \%, 93.94 \%$, and $96.97 \%$, respectively. The continence rates in Group B were $10.94 \%, 14.06 \%, 37.50 \%$, and $71.88 \%$, respectively. The continence rate of Group A was significantly higher after surgery compared with Group B $(P<0.001)$. Maximum flow rates before and after the "Sandwich" procedure for 12 months were 13.2 $\pm 2.8 \mathrm{~m} / \mathrm{s}$ and $15.4 \pm 3.6 \mathrm{~m} / \mathrm{s}$, respectively $(P=0.034)$. In addition, residual volumes before and after the "Sandwich" procedure for 12 months were $15(0-20) \mathrm{mL}$ and $0(0-12.5) \mathrm{mL}$, respectively $(P=0.107)$.

Conclusion: Our prospective study confirms that the "Sandwich" technique of the total urethral reconstruction is safe and feasible. It also very possibly takes the significant advantage in early recovery of urinary continence after LRP. However, multicenter, randomized controlled large sample randomized controlled trials are needed to further confirm this final conclusion.

Keywords: Sandwich, urethral reconstruction, laparoscopic radical prostatectomy, urinary incontinence, urodynamic tests

\section{Introduction}

Prostate cancer is one of the most common cancers for men in the world, ${ }^{1}$ and radical prostatectomy is an optimal standard method for treating patients who suffer from localized prostate cancer. With the increasing aging of the population in China, the number of patients diagnosed with prostate cancer has been continuously increasing recently. ${ }^{2}$ With the development of minimally invasive techniques, LRP or robotassisted radical prostatectomy (RALP) has become the preferred surgical method in
Department of Urology, National Cancer Center/National Clinical Research Center for Cancer/Cancer Hospital, Chinese Academy of Medical Sciences and Peking Union Medical College, No. 17, Panjiayuan South Li, Chaoyang, Beijing, I0002I, People's Republic of China

Email xingnianzeng@I26.com 
many large medical centers. ${ }^{3}$ These minimally invasive techniques have also achieved good oncological outcomes. ${ }^{4,5}$ Therefore, sexual function and urinary continence after LRP or RALP may be of most concern for both the surgeon and patient. Since the anatomic approach has been introduced to radical prostatectomy (RP), the surgeon has done many surgical innovations to improve urinary continence and sexual function recovery, such as intrafascial sparing nerve technique ${ }^{6,7}$ and urethral reconstruction technique. ${ }^{8,9}$ In our study, we designed a prospective study to further verify the effectiveness, especially improving the continence at an early stage, of our "Sandwich" technique of the total urethral reconstruction.

\section{Methods}

All patients who have undergone LRP in our institution during the period from October 2017 to December 2018 were recruited for this prospective study. This study included 130 patients, who were divided into two groups. Sixty-six patients in Group A received LRP with "Sandwich" technique of urethra reconstruction, while the remaining 64 patients in Group B did not adopt this reconstruction technique.

The preoperative protocol included the measurement of BMI, prostate-specific antigen (PSA) before the operation, ultrasound for prostate volumes, the examination of the digital rectum (DRE), magnetic resonance imaging (MRI) of the prostate, transrectal prostate biopsy using ultrasound, and bone scintigraphy. The exclusion criteria of the study included the expectation of life being less than 10 years and suffering from metastatic or locally advanced prostate cancer. The study was approved by the Research Ethics Committee of Cancer Hospital, Chinese Academy of Medical Sciences and Beijing Chaoyang Hospital, Capital Medical University. All the patients in this study provided written informed consent.

\section{Surgical Technique}

Under general anesthesia, an extraperitoneal working space was first created via a five-port approach, as described by Stolzenburg et al, ${ }^{10}$ and the prostate, bladder, and endopelvic fascia were exposed. Then the endopelvic fascia was bilaterally incised and dissected towards the apex of prostate at the beginning. Next the puboprostatic ligament was cut and the dorsal venous complex (DVC) was ligated using a $15 \mathrm{~cm}$ and barbed thread and then the bladder neck was carefully transected and preserved. After the dissection of seminal vesicles from vas deferens on both sides, it was easy to incise Denonvillier's fascia and dissect it down to the apex of the prostate. Next the vascular pedicle (including NVB) was preserved to the maximum extent for selected patients through Hem-O-Lok clip and the cold scissor was used for incising the prostatic fascia sharply and cutting the urethra in the external urethral sphincter center and the prostate apex while the puboprostatic ligaments were kept. Bilateral pelvic lymphadenectomy was carried out for all patients, and the specimens of prostate and lymph nodes were placed in a specimen bag and eventually removed through an umbilical incision.

In Group A, the first step of total urethral reconstruction of the "Sandwich" was started with the reconstruction of the posterior wall via reconstructing Denonvillier's fascia in two layers. In the first layer, the upper end of the severed fascia was sutured with MDR (median dorsal ralph) (Figure 1); and, in the second layer, MDR was sutured with the bladder wall at the back of the posterior lip of the bladder neck (Figure 2).

Urethrovesical anastomosis was the second step of the reconstruction. A running urethrovesical anastomosis using an absorbable barbed suture was performed with the posterior wall suturing from 4 o'clock to 8 o'clock and the front wall suturing from 3 o'clock to 9 o'clock (Figure 3). The first suture was performed from outside-inside in the right posterior of the neck of the bladder, and this suture was put on the urethral stump section from inside to outside
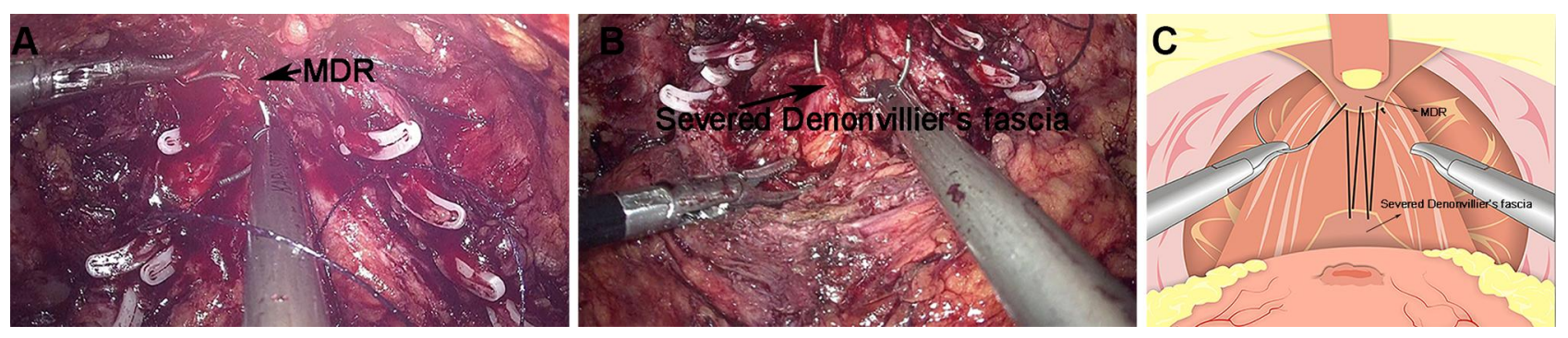

Figure I The first layer of the reconstruction of the posterior wall of total urethral reconstruction of "Sandwich". (A) The structural location of the medial dorsal raphe (MDR) during the operation. (B) The structural location of the severed end of Denonvillier's fascia. (C) The severed Denonvillier's fascia was sutured with MDR. 

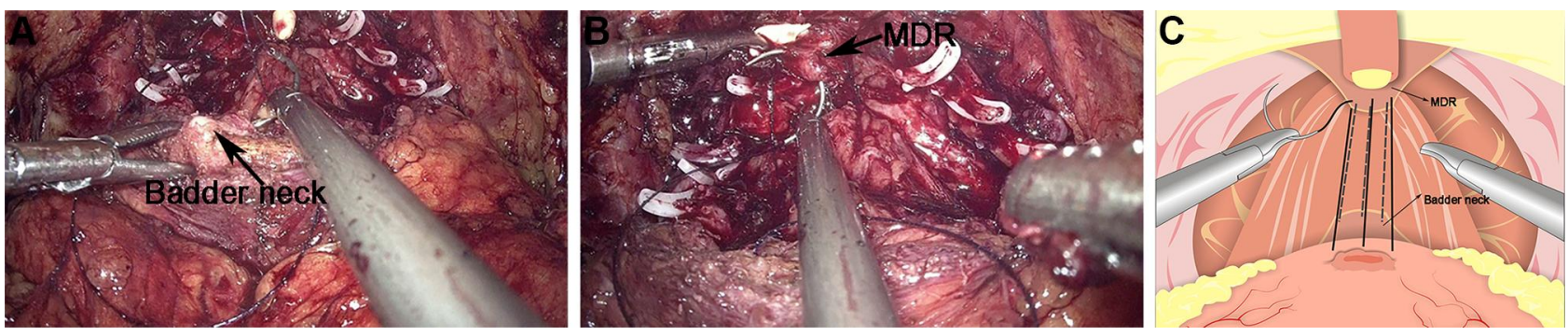

Figure 2 The second layer of the reconstruction of the posterior wall of total urethral reconstruction of "Sandwich". (A) The structural location of the MDR during the operation. (B) The structural location of the posterior lip of the ladder neck. (C) MDR was sutured with the bladder wall at the back of the posterior lip of the bladder neck.
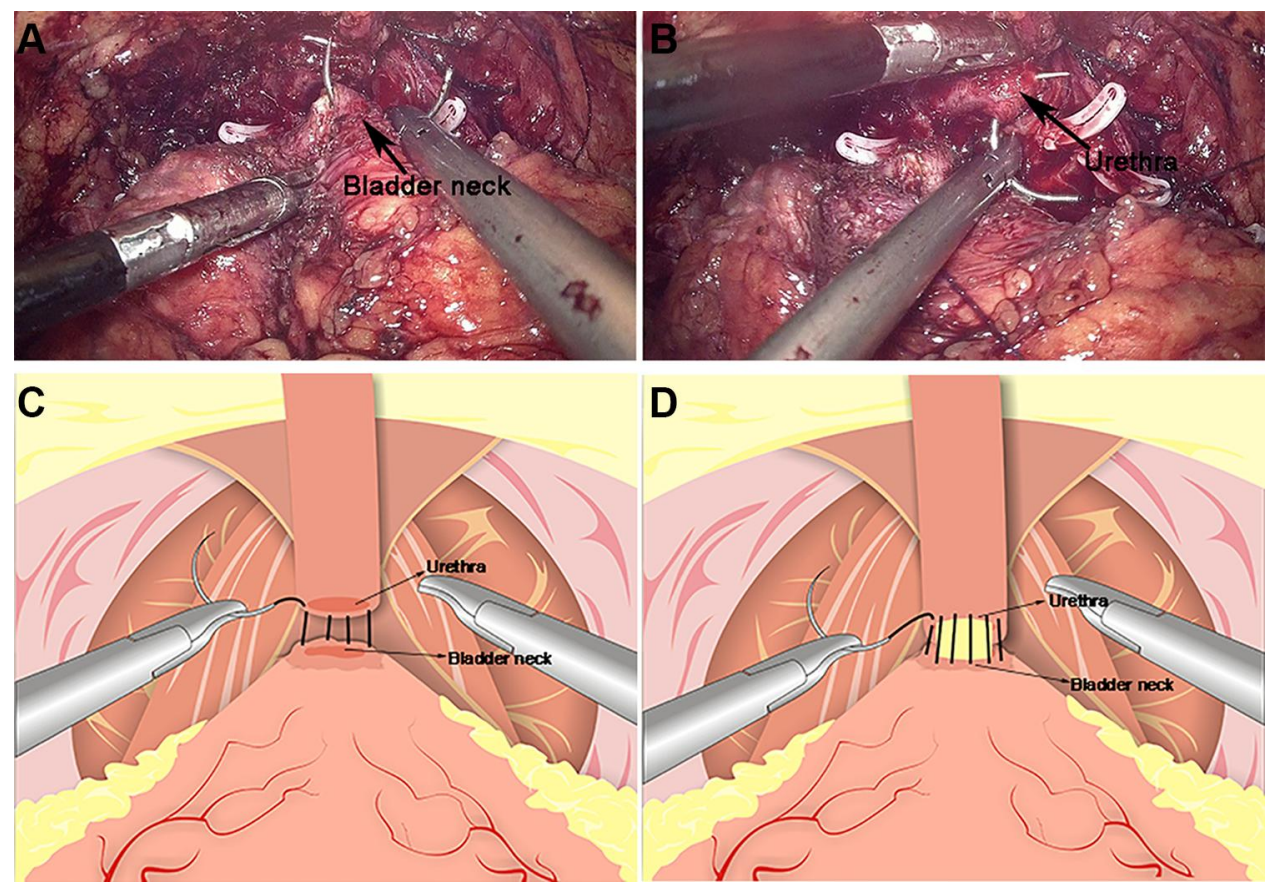

Figure 3 Anastomosis of bladder and urethra of total urethral reconstruction of the "Sandwich". (A) The structural location of the bladder neck during the operation. (B) The structural location of the urethra. (C) A running urethrovesical anastomosis was performed with the posterior wall suturing from 4 o'clock to 8 o'clock. (D) A urethrovesical anastomosis was performed with the front wall suturing from 3 o'clock to 9 o'clock.

correspondingly. Later, the processes from outside-inside at the neck of the bladder and inside-outside at the urethral stump were in the left of the neck of the bladder repeatedly. The 18F Silastic Foley catheter had been inserted into the bladder gently. When it had been sutured continuously around the neck of the bladder with the order from left to right, the urethrovesical anastomosis had been finished.

The third step was the reconstruction of the anterior wall of the urethra, including reattaching the arcus tendinous and pubicoprostatic ligament to the bladder neck (Figure 4). A barbed absorbable suture was applied to approximate the remained arcus tendinous and distal triangular plate anterior to the urethra, which included the residual endopelvic fascia rhabdosphincter, puboprostatic ligaments and dorsal venous complex to the neck of the bladder. In Group B, the urethral was reconstructed in the second step in Group A only.

According to Expanded Prostate Cancer Index Composite for Clinical Practice (EPIC-CP), ${ }^{11}$ patients who achieved total control or occasional dribbling or required 0 or 1 pad were considered continent; those who have normal physical activity (walking) requiring 2-3 pads/day were defined as mildly incontinent (stress incontinence); the patients who required for more than 3 pads/day were considered as incontinent.

\section{Statistical Analysis}

A parametric continuous variable was presented as a mean \pm standard deviation; non-parametric continuous variable was shown in a median and interquartile range (IQR); the $t$-tests 

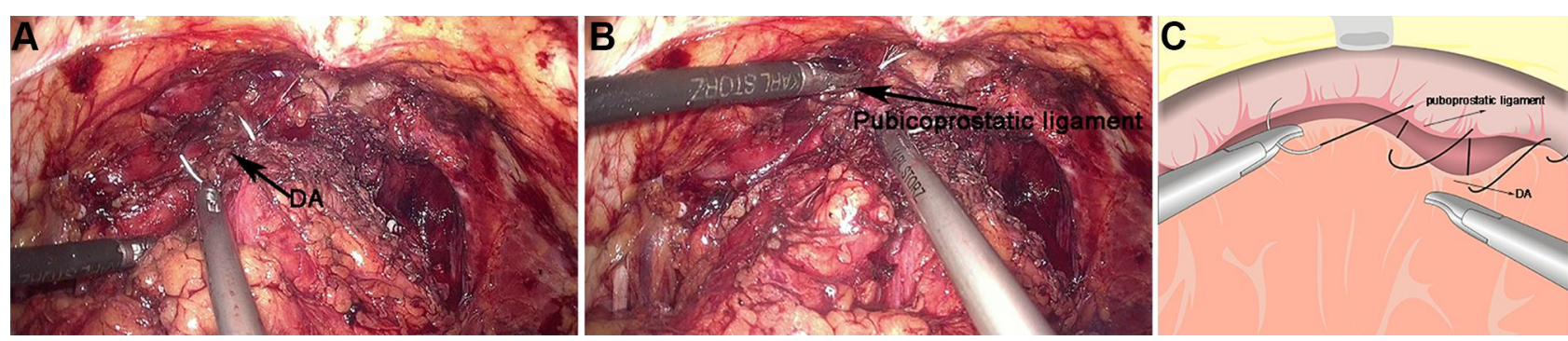

Figure 4 The reconstruction of the anterior of total urethral reconstruction of the "Sandwich". (A) The structural location of the detrusor apron (DA) during the operation. (B) The structural location of the pubicoprostatic ligament. (C) The pubicoprostatic ligament is sutured with the bladder wall behind the anterior lip of the bladder neck (equivalent to the position of DA).

of these two groups were used for comparing numerical variables. SPSS 21.0 Software (IBM Corp, Armonk, NY) was used for processing the data. The statistical significance was $P<0.05$.

\section{Results}

Neither conversion nor re-intervention was found in these 130 cases of Group A and Group B. Similar perioperative data were found in these two groups (Table 1). The total operative time, blood loss, duration of the catheter, and post-operative hospital stay were similar to each other in both groups (Table 2), but the time of urethral anastomosis in Group A was longer than that in the other group $(P<0.01)$. The pathological stages and positive surgical margin (PSM) rates with a positive value in these two groups were almost the same as each other (Table 3). Table 4 shows the postoperative urinary continence rate of both groups. In Group A, $54.5 \%$ of the patients were considered as continent at 2 weeks. Group B was $10.9 \%$. Both groups' differences were significant statistically $(P<0.01)$. At 4 weeks, the percentages of patients who were continent in both groups were $83.3 \%$ and $14.1 \%$, which were statistically significantly

Table I Baseline Characteristics of the Patients

\begin{tabular}{|l|l|l|l|}
\hline & Group A & Group B & P-value \\
\hline Patient, $\mathrm{n}$ & 66 & 64 & \\
\hline Age, years & $67.74 \pm 5.20$ & $66.22 \pm 6.50$ & 0.255 \\
\hline BMl, kg/m² & $25.35 \pm 3.37$ & $24.60 \pm 3.18$ & 0.06 \\
\hline Prostate weight, g & $46.59 \pm 20.85$ & $41.41 \pm 14.95$ & 0.697 \\
\hline $\begin{array}{c}\text { Biopsy Gleason score, } \mathrm{n}(\%) \\
\leq 6\end{array}$ & $15(22.73)$ & $15(23.44)$ & 0.904 \\
7 & $28(42.42)$ & $29(45.3 \mathrm{I})$ & \\
$\geq 8$ & $23(34.85)$ & $20(31.25)$ & \\
\hline
\end{tabular}

Note: Data is represented as mean $\pm S D$ or $n(\%)$, unless otherwise stated. Abbreviation: BMl, body mass index. different $(P<0.01)$. The continence rates at 8 and 12 weeks in Group A were $93.9 \%$ and $96.9 \%$, respectively; and the continence rates in Group B were $37.5 \%$ and $71.9 \%$, respectively. The continence rates were significant $(P<0.01$, respectively). We followed up the patients who had urodynamic tests and found that maximum flow rates before and after the "Sandwich" procedure for 12 months were 13.2 $\pm 2.8 \mathrm{~m} / \mathrm{s}$ and $15.4 \pm 3.6 \mathrm{~m} / \mathrm{s}$, respectively, which have a statistical difference $(P=0.034)$. In addition, residual volumes before and after "Sandwich" procedure for 12 months were $15(0-20) \mathrm{mL}, 0(0-12.5) \mathrm{mL}$, which have no statistical difference $(P=0.107)$.

\section{Discussion}

Compared with open surgical technique, laparoscopic technique takes the advantage of the hospitalization,

Table 2 Operative Date of the Patients

\begin{tabular}{|l|l|l|l|}
\hline & Group A & Group B & P-value \\
\hline $\begin{array}{l}\text { Operative time, minutes } \\
\text { (range) }\end{array}$ & $120.64 \pm 58.36$ & $106.06 \pm 45.07$ & 0.094 \\
\hline $\begin{array}{l}\text { Reconstruction Time, } \\
\text { minutes (range) }\end{array}$ & $23.49 \pm 4.72$ & $20.16 \pm 5.75$ & $<0.01$ \\
\hline Estimated blood loss, mL & $120.77 \pm 88.86$ & $108.41 \pm 75.77$ & 0.444 \\
\hline Hospital length of stay, days & $8.14 \pm 3.75$ & $7.47 \pm 3.66$ & 0.079 \\
\hline Duration of Catheter, days & $11.80 \pm 5.68$ & $10.34 \pm 5.07$ & 0.094 \\
\hline $\begin{array}{l}\text { Procedure with sparing } \\
\text { nerve, } n \text { (\%) } \\
\text { Bilateral nerve sparing } \\
\text { Unilateral nerve sparing }\end{array}$ & $\begin{array}{l}9(13.64) \\
\text { Non-nerve sparing }\end{array}$ & $\begin{array}{l}51(77.27) \\
4(6.25)\end{array}$ & $\begin{array}{l}4(6.25) \\
56(87.5)\end{array}$ \\
\hline $\begin{array}{c}\text { Complications, n (\%) } \\
\text { Complications grade I } \\
\text { Complications grade II }\end{array}$ & $\begin{array}{l}5(7.58) \\
0(0)\end{array}$ & $\begin{array}{l}5(7.81) \\
1(1.56)\end{array}$ & 0.267 \\
\hline
\end{tabular}

Note: Data is represented as mean $\pm S D$ or $n(\%)$, unless otherwise stated. 
Table 3 Postoperative Data of the Patients

\begin{tabular}{|c|c|c|c|}
\hline & Group A & Group B & $P$-value \\
\hline Pathologic stage, $\mathrm{n}$ & & & 0.771 \\
\hline PTO & 0 & 0 & \\
\hline PT2a & 4 & 5 & \\
\hline $\mathrm{pT} 2 \mathrm{~b}$ & 12 & 10 & \\
\hline PT2c & 41 & 43 & \\
\hline pT3 & 9 & 6 & \\
\hline pT4 & 0 & 0 & \\
\hline PSM rate, n (\%) & $21(31.82)$ & $13(20.31)$ & 0.136 \\
\hline $\begin{array}{l}\text { Gleason sore-final specimen, } \\
\mathrm{n}(\%)\end{array}$ & & & 0.675 \\
\hline$\leq 6$ & $10(15.16)$ & $10(15.62)$ & \\
\hline 7 & $40(60.6 I)$ & $35(54.69)$ & \\
\hline$\geq 8$ & $16(24.24)$ & $19(29.69)$ & \\
\hline
\end{tabular}

Note: Data is represented as mean $\pm S D$ or $n(\%)$, unless otherwise stated. Abbreviation: PSM, positive surgical margin.

Table 4 Postoperative Urinary Continence Rate of Both Groups

\begin{tabular}{|l|l|l|l|}
\hline Continence Rates n (\%) & Group A & Group B & P-value \\
\hline 2 weeks & $36(54.55)$ & $7(10.94)$ & $<0.01$ \\
\hline 4 weeks & $55(83.33)$ & $9(14.06)$ & $<0.01$ \\
\hline 8 weeks & $62(93.94)$ & $24(37.50)$ & $<0.01$ \\
\hline 12 weeks & $64(96.97)$ & $46(71.88)$ & $<0.01$ \\
\hline
\end{tabular}

Note: Data is represented as n (\%), unless otherwise stated.

blood loss, postoperative complications, and recovery time. ${ }^{12}$ However, urinary incontinence after LRP may significantly impact patients' quality-of-life. They may have feelings of low self-esteem, anxiety, and depression. ${ }^{13}$ UI after LRP is mainly from urethral sphincter deficiency or detrusor overactivity. ${ }^{14}$ Therefore, the preservation of many anatomic structures of the prostate and the way of urethral reconstruction are important factors for the recovery of urine control and even sexual function. ${ }^{15}$

The anatomical structures critical to continence were affected to different extents after surgery, which encompassed the muscular components, the urethral components, and the detrusor components. ${ }^{16}$ Muscular components included the neck of the bladder, internal and external rhabdosphincter, membranous urethra, and puboperinealis as well as pelvic floor muscles, which support structures of the sphincteric complex, such as arcustendinosus, puboprostatic ligament, detrusor apron, denonvilliers' fascia, and pelvic bones as well as pelvic floor levator ani muscle, and ligaments. The urethral components involved in sphincteric incompetence, which might be caused by the length of the short urethral stump, neural innervations loss, damage to muscle and supporting tissue loss around the potential. The detrusor components involved in denovo bladder instability, detrusor over-activity or hypocontractility due to damage to nerve or compliance of diminished bladder. ${ }^{17}$

The preservation of anatomical structures, such as the endopelvic fascia, the detrusor apron nerve through intrafascial dissecting, and suitable length of urethral membrane contributed to early recovery of urinary continence. ${ }^{18}$ It was shown that nerve-preserving bilateral processes can retain the function of motor control of the urethral sphincter, thereby improving postoperative urinary control. ${ }^{19}$ The detrusor apron, extended from the bladder's interior wall with direct continuity of pubis, had aroused widespread concern recently. The detrusor apron was divided into an anterior, a middle, and a posterior layer anterior to the prostate. The anterior layer passes to the decussated pubococcygeal fibersposteriorly, defuses anteroinferior, and anchors onto the posterior surface of the pubic bone. The middle layer is loose, which connects with the fascial sheath of the dorsal venous complex (DVC). Finally, the posterior layer encloses and extends into the prostate to form the anterior fibromuscular stroma (AFMS) of the prostate..$^{20,21}$ The urethra is also an important anatomical structure with preservation for continence essentially. Generally, the long urethral stump with lateral supporting tissue is critical to reducing the incontinence risk after the operation. It is shown that the membranous urethra retracts proximally immediately after RP while the urethral stump retracts toward the bladder after the urethrovesical anastomosis. Such processes have a negative impact on the functions of the urethral sphincter and the urethral closure pressure. But with time, such changes can be recovered to their preoperative location, and the urethral closure pressure and urinary continence can be recovered. $^{22}$

At present, there are a variety of urethral reconstruction techniques, such as posterior wall reconstruction (represented by Roccostitch) and bladder neck anterior wall suspension and total reconstruction of the vesicourethral anastomosis. ${ }^{23,24}$ In our study, the construction of the "Sandwich" urethra was started with posterior reconstruction. This surgical step mainly targets minimizing urethrosphincteric sliding after RP for supporting VUA, descending the neck of the bladder to the urethral stump 
closely, and providing enough contraction fulcrum for the rhabdosphincter. It was reported that the rhabdosphincter reconstruction at a posterior position led to continence recovery at an early stage and reduced anastomotic leakage. ${ }^{25}$

After urethral anastomosis, the anterior suspension was started, which is another important step in sandwich reconstruction. Fixation of the neck of the bladder to the pubis is a proposed technique for anterior suspension through putting a bulbourethral stitch or suspending the neck of the bladder along the VUA with/without the ligated DVC to the pubic symphysis. According to some authors, the continence rates were $53 \%, 73 \%$, and $100 \%$ at 1,3 , and 6 months, respectively, compared with the control group rates of $20 \%, 47 \%$, and $83 \%$, respectively. ${ }^{26}$ In a meta-analysis, using this bimodal reconstruction increases short-term (defined as 1-12 weeks) and long-term (up to a year after the operation) urinary continence rates. In our study, comparison between the posterior reconstruction and nonreconstruction, patient's outcomes of the urinary continence are $49 \%$ vs $24 \%$ at 3 months, and $92 \%$ vs $79 \%$ at 1 -year post-RP. Once again, it shows the superiority of using sandwich reconstruction technology. It shows once again that the "Sandwich" technique of the total urethral reconstruction is beneficial to the recovery of early urine continence without affecting urodynamic parameters after RP and increasing complications, such as bleeding, positive margins, and prolonged surgical time.

\section{Conclusion}

Our prospective study confirms that the "Sandwich" technique of the total urethral reconstruction is safe and feasible. It also very possibly takes the significant advantage in early recovery of urinary continence after LRP. However, multicenter, randomized controlled large sample randomized controlled trials are needed to further confirm this final conclusion.

\section{Abbreviations}

RALP, robot-assisted radical prostatectomy; LRP, laparoscopic radical prostatectomy; NVB, neurovascular bundle; $\mathrm{RP}$, radical prostatectomy; PSA, prostate-specific antigen; EDR, an examination of the digital rectum; MRI, magnetic resonance imaging; DVC, dorsal venous complex; DF, Denonvilliers' fascia; IQR, interquartile range; UI, urinary incontinence; AFMS, anterior fibromuscular stroma; BMI, body mass index; PSM, positive surgical margin; MDR, median dorsal ralph.

\section{Clinical Trial}

The trial Registration Number is ChiCTR-IPR-15005903. The name of the registry is "Total Reconstruction" of the Urethrovesical Anastomosis Contributes to Early Urinary Continence in Laparoscopic Radical Prostatectomy. The date of registration is October 19, 2017. The URL of the trial registry record is http://www.medresman.org.cn/uc/ project/projectedit.aspx?proj=2912.

\section{Data Sharing Statement}

Data used and/or analyzed in the current research can be obtained from the corresponding author on reasonable request.

\section{Ethics Approval and Consent to Participate}

This trial was conducted in accordance with the Declaration of Helsinki. The study was approved by Research Ethics Committee of Cancer Hospital, Chinese Academy of Medical Sciences and Beijing Chaoyang Hospital, Capital Medical University. All the patients in this study provided written informed consent. Each enrolled patient provided written informed consent.

\section{Consent for Publication}

Informed consent was obtained from the patients.

\section{Author Contributions}

All authors made substantial contributions to the conception and design, acquisition of data, or analysis and interpretation of data; took part in drafting the article or revising it critically for important intellectual content; agreed to submit to the current journal; gave final approval of the version to be published; and agree to be accountable for all aspects of the work.

\section{Funding}

This research was financially supported by the Capital Science and Technology Leading Talent Project (Project number: Z181100006318007).

\section{Disclosure}

The authors declare that they have no competing interests. 


\section{References}

1. Resnick MJ, Lacchetti C, Bergman J, et al. Prostate cancer survivorship care guideline: American society of clinical oncology clinical practice guideline endorsement. J Clin Oncol. 2015;33(9):1078-10 85. doi:10.1200/JCO.2014.60.2557

2. Qi D, Wu C, Liu F, et al. Trends of prostate cancer incidence and mortality in Shanghai, China from 1973 to 2009. Prostate. 2015;75 (14):1662-1668. doi:10.1002/pros.23046

3. Rassweiler J, Hruza M, Teber D, et al. Laparoscopic and roboticassisted radical prostatectomy-critical analysis of the results. Eur Urol. 2006;49(4):612-624. doi:10.1016/j.eururo.2005.12.054

4. Luzzago S, Rosiello G, Pecoraro A, et al. Contemporary rates and predictors of open conversion during minimally invasive radical prostatectomy for nonmetastatic prostate cancer. $J$ Endourol. 2020;34(5):600-607. doi:10.1089/end.2020.0074

5. Stolzenbach LF, Knipper S, Mandel P, et al. Oncological outcomes of pathologically organ-confined, lymph node-positive prostate cancer after radical prostatectomy. Urol Oncol. 2020. doi:10.1016/j.urolonc. 2020.10.010

6. Tewari A, Peabody JO, Fischer M, et al. An operative and anatomic study to help in nerve sparing during laparoscopic and robotic radical prostatectomy. Eur Urol. 2003;43(5):444-454. doi:10.1016/S03022838(03)00093-9

7. Fromont G, Baumert H, Cathelineau X, et al. Intraoperative frozen section analysis during nerve sparing laparoscopic radical prostatectomy: feasibility study. J Urol. 2003;170(5):1843-1846. doi:10.1097/ 01.ju.0000092081.71167.34

8. Liao X, Qiao P, Tan Z, et al. "Total reconstruction" of the urethrovesical anastomosis contributes to early urinary continence in laparoscopic radical prostatectomy. Int Braz J Urol. 2016;42(2):215-222. doi:10.1590/S1677-5538.IBJU.2014.0666

9. Vis AN, van der Poel HG, Ruiter AEC, et al. Posterior, anterior, and periurethral surgical reconstruction of urinary continence mechanisms in robot-assisted radical prostatectomy: a description and video compilation of commonly performed surgical techniques. Eur Urol. 2019;76(6):814-822. doi:10.1016/j.eururo.2018.11.035

10. Stolzenburg JU, Do M, Pfeiffer H, et al. The endoscopic extraperitoneal radical prostatectomy (EERPE): technique and initial experience. World J Urol. 2002;20(1):48-55. doi:10.1007/s00345002-0265-4

11. Lourenço DB, Amaral BS, Alfer-Junior W, et al. Portuguese version of the expanded prostate cancer index composite for clinical practice (EPIC-CP): psychometric validation and prospective application for early functional outcomes at a single institution. BMC Urol. 2020;20 (1):163. doi:10.1186/s12894-020-00734-y

12. Guillonneau B, EI-Fettouh H, Beaument $\mathrm{H}$, et al. Laparoscopic radical prostatectomy: oncological evaluation after 1,000 cases a Montsouris Institute. J Urol. 2003;169(4):1261-1266. doi:10.10 97/01.ju.0000055141.36916.be

13. Da Mata LR, de Carvalho EC, Gomes CR, et al. Post-operative self-efficacy and psychological morbidity in radical prostatectomy. Rev Lat Am Enfermagem. 2015;23(5):806-813. doi:10.1590/01041169.0456 .2618

Cancer Management and Research

\section{Publish your work in this journal}

Cancer Management and Research is an international, peer-reviewed open access journal focusing on cancer research and the optimal use of preventative and integrated treatment interventions to achieve improved outcomes, enhanced survival and quality of life for the cancer patient.
14. Patel MI, Yao J, Hirschhorn AD, et al. Preoperative pelvic floor physiotherapy improves continence after radical retropubic prostatectomy. Int J Urol. 2013;20(10):986-992. doi:10.1111/iju.12099

15. Theissen L, Preisser F, Wenzel M, et al. Very early continence after radical prostatectomy and its influencing factors. Front Surg. 2019;25 (6):60. doi:10.3389/fsurg.2019.00060

16. Walz J, Burnett AL, Costello AJ, et al. A critical analysis of the current knowledge of surgical anatomy related to optimization of cancer control and preservation of continence and erection in candidates for radical prostatectomy. Eur Urol. 2010;57(2):179-192. doi:10.1016/j.eururo.2009.11.009

17. Walz J, Epstein JI, Ganzer R, et al. A critical analysis of the current knowledge of surgical anatomy of the prostate related to optimisation of cancer control and preservation of continence and erection in candidates for radical prostatectomy: an update. Eur Urol. 2016;70 (2):301-311. doi:10.1016/j.eururo.2016.01.026

18. Laucirica O, Gomez E, Hajianfar R, Vilanova JC, Muniesa M. Complete puborectalis, puboperinealis muscle and urethral rhabdomyosphincter preservation in laparoscopic radical prostatectomy: anatomical landmarks to achieve early urinary continence. Int J Urol. 2020;27(6):525-536. doi:10.1111/iju.14228

19. Nandipati KC, Raina R, Agarwal A, et al. Nerve-sparing surgery significantly affects long-term continence after radical prostatectomy. Urology. 2007;70(6):1127-1130. doi:10.1016/j.urology.2007.07.042

20. Xu Z, Chapuis PH, Bokey L, et al. Nature and architecture of the puboprostatic ligament: a macro- and microscopic cadaveric study using epoxy sheet plastination. Urology. 2017;110:263.e1-263.e8. doi:10.1016/j.urology.2017.08.018

21. Myers RP. Detrusor apron, associated vascular plexus, and avascular plane: relevance to radical retropubic prostatectomy-anatomic and surgical commentary. Urology. 2002;59(4):472-479. doi:10.1016/ S0090-4295(02)01500-5

22. Kadono Y, Nohara T, Kawaguchi S, et al. Investigating the mechanism underlying urinary continence recovery after radical prostatectomy: effectiveness of a longer urethral stump to prevent urinary incontinence. BJU Int. 2018;122(3):456-462. doi:10.1111/bju.14181

23. Grasso AA, Mistretta FA, Sandri M, et al. Posterior musculofascial reconstruction after radical prostatectomy: an updated systematic review and a meta-analysis. BJU Int. 2016;118(1):20-34. doi:10.11 $11 /$ bju. 13480

24. Tewari A, Jhaveri J, Rao S, et al. Total reconstruction of the vesico-urethral junction. BJU Int. 2008;101(7):871-877. doi:10.111 1/j.1464-410X.2008.07424.x

25. Rocco BMC, Bianchi G. Editorial comment on: three-layer two-step posterior reconstruction using peritoneum during robot-assisted radical prostatectomy to improve recovery of urinary continence: a prospective comparative study by Ogawa et al. $J$ Endourol. 2017;31(12):1258. doi:10.1089/end.2017.0823

26. Noguchi M, Kakuma T, Suekane S, et al. A randomized clinical trial of suspension technique for improving early recovery of urinary continence after radical retropubic prostatectomy. BJU Int. 2008;102(8):958-963. doi:10.1111/j.1464-410X.2008.07759.x 